\title{
Multipath fading effects on carrier recovery of BPSK signal in digital radio
}

\author{
G.-S. Liu, MSc \\ C.-H. Wei, PhD
}

Indexing terms: Carrier recovery, Digital radio, BPSK, Intersymbol interference, Multipath fading

Abstract: Tracking performance of the $I-Q$ carrier recovery loop for BPSK signal in bandlimited channel with multipath interference is analysed. The multipath fading channel is characterised by the Rummler's model (a simplified three-ray model) for line-of-sight digital radio system. A closed-form expression for the phaseerror variance related to the multipath interference, intersymbol interference and channel noise is derived. Excess phase offset induced from the multipath interference is also examined. The closed-form expression of the jitter variance can be evaluated numerically to assess the degradation of synchroniser performance. Asymptotic limits of the performance are also discussed.

\section{Introduction}

Carrier recovery is crucial for coherent detection in digital communication systems. It recovers the carrier phase from noise-corrupted and severely interfered signals. The main impairments which degrade the performance of a carrier synchroniser are self noise, cyclic slipping, channel noise, intersymbol interference (ISI) and sometimes multipath interference. Among these impairments, the ISI effects on the carrier recovery loop have been well discussed in the literature [1-6]. Hinedi and Lindsey [6] have derived a closed-form expression for the phase error variance due to ISI. Most HF radio channels in which fading is encountered are basically line-of-sight (LOS) communication links with multipath components arising from secondary reflections, or signal paths from surrounding terrain. In such channels the number of multipath components is small, so the channel can be modelled by a two- or three-ray model [7]. The statistics of the model parameters for two or three rays can be determined empirically through the field measurement data. The properties of the three-ray propagation models are studied in References 14 and 15. Channel models based on three rays have been proposed in References 11 and 16-19.

In this paper, by using the simplified three-ray multipath model (Rummler's model [11]), the statistical characteristics of the phase error for the $I / Q$ carrier-recovery

\section{(C) IEE, 1993}

Paper 9683I (E8), received 25th January and in revised form 1st June 1993

The authors are with the Institute of Electronics and Center for Telecommunications Research, National Chiao-Tung University, Hsinchu, Taiwan, Republic of China

IEE PROCEEDINGS-I, Vol. 140, No. S, OCTOBER 1993 loop in the multipath channel are obtained in several closed-form expressions. Asymptotic bounds of the performance and numerical examples in terms of the channel characteristics are also examined.

\section{Formulation}

A BPSK signal can be written in the following general form

$$
\mathrm{s}(t)=\sqrt{(2 P) m(t) \sin \left(\omega_{0} t+\theta\right)}
$$

where

$$
m(t)=\sum_{k=-\infty}^{\infty} a_{k} p(t-k T)
$$

represents the data modulation on the signal after bandlimiting filtering. In eqn. 1, the carrier phase is denoted by $\theta, a_{k}$ is chosen from symbol set $\{+1,-1\}$ with equal probability and $p(t)=g(t) * h(t)$, where * represents the convolution operation. Here, $g(t)$ stands for the signalling pulse and $h(t)$ is the impulse response of the bandlimited channel. The signal encounters ISI effects due to the bandlimitation.

In radio communications, the transmitted signal may be reflected by obstacles and the received signal is diffused with the reflected interferences from multiple paths. Owing to the frequency selective fading it will induce another source of ISI on the received signal, in addition to that by bandlimitation. Dispersion due to multipath propagation degrades digital transmission via the generation of ISI. In this paper, these two different sources of ISI effects are considered simultaneously on the performance of the carrier tracking loop for BPSK signal.

A simplified three-ray model based on channel measurement performed on microwave LOS radio channels has been proposed by Rummler [11-13]. The low-pass equivalent transfer function of the three-ray model with fixed delay parameters can be written

$$
C(j \omega)=\alpha\left\{1-\beta e^{-j\left(\omega-\omega_{F) \tau}\right.}\right\} \equiv \alpha\{1-\beta D(j \omega)\}
$$

where the frequencies $\omega$ and $\omega_{\mathrm{F}}$ are measured from the central frequency $\omega_{0}$. In eqn. 3 , parameter $\alpha$ is the overall attenuation factor, $\beta$ is a shape parameter due to the multipath component, $\omega_{F}$ is the angular frequency of the

This work was supported by the National Science Council, Republic of China, under grant NSC820404-E009-122. The authors would like to thank the referees for their valuable comments in revising the manuscripts. 
fade minimum (or referred to as notch frequency), and $\tau$ is the relative time delay between the direct and the multipath components. The transfer function can be interpreted as the response of a channel that provides a direct transmission path with amplitude factor $\alpha$ and a secondary path with relative strength $\beta$, at a fixed delay $\tau$ and a phase offset $\omega_{F} \tau+\pi$ at the central frequency (phase $\pi$ results in a minus sign in eqn. 3). Note that eqn. 3 has the appearance of a two-path response. This response can be viewed as arising from three different paths. Among these three components, the direct path signal is unfaded, and the second path is similar in strength and close enough in delay to the first one such that their composite response is constant (here denoted by $\alpha$ ). The third path at relative delay $\tau$ and relative strength $\beta$ provides the frequency shaping of $C(j \omega)$. The block diagram of the simplified three-ray model is shown in Fig. 1, where $D(j \omega)$ can be viewed as an allpass transfer function with linear phase $\phi_{D}(\omega)=-\left(\omega-\omega_{F}\right) \tau$ and

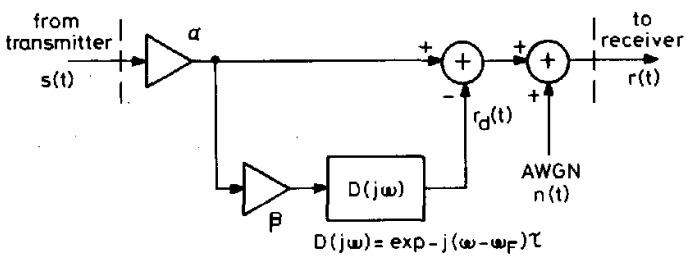

Fig. 1 Simplified three-ray model for multipath channel

flat magnitude response $|D(j \omega)|=1$. Assume that the envelope $m(t)$ of the signal varies slowly, the output signal of the lower arm can be approximated by

$$
r_{d}(t) \approx \alpha \beta\left\{\sqrt{ }(2 P) m\left(t-\tau_{g}\right) \sin \left(\omega_{0}\left(t-\tau_{p}\right)+\theta\right)\right\}
$$

where $\tau_{g}$ and $\tau_{p}$ represent the group delay and the phase delay of $D(j \omega)$ at the neighbourhood of carrier frequency $\omega_{0}$, respectively.

$$
\begin{aligned}
& \tau_{\theta} \equiv-\left.\frac{d \phi_{D}\left(\omega-\omega_{0}\right)}{d \omega}\right|_{\omega=\omega_{0}}=\tau \\
& \tau_{p} \equiv-\left.\frac{\phi_{D}\left(\omega-\omega_{0}\right)}{\omega}\right|_{\omega=\omega_{0}}=-\frac{\omega_{F}}{\omega_{0}} \tau
\end{aligned}
$$

Therefore, by using eqns. 4 and 5 , the received signal at the channel output can be written

$$
\begin{aligned}
r(t)= & \alpha \sqrt{ }(2 P) m(t) \sin \left(\omega_{0} t+\theta\right)-\alpha \beta \sqrt{ }(2 P) m(t-\tau) \\
& \times \sin \left(\omega_{0} t+\omega_{F} \tau+\theta\right)+n(t)
\end{aligned}
$$

The first term represents the signal component, the second term is viewed as the multipath interference and the third term is the channel noise. The channel noise $n(t)$ considered here is a white Gaussian noise, expressed by

$$
n(t)=\sqrt{ }(2)\left[n_{c}(t) \cos \left(\omega_{0} t+\varphi\right)-n_{s}(t) \sin \left(\omega_{0} t+\varphi\right)\right]
$$

where $\varphi$ is the noise phase. The in-phase and quadrature components of the noise are assumed to be statistically independent, stationary white Gaussian noise processes with (two-sided) power spectral density $N_{0} / 2$ watt $/ \mathrm{Hz}$. These noise components can be rewritten from eqn. 7 as

$$
n(t)=\sqrt{ }(2)\left[N_{c}(t) \cos \left(\omega_{0} t+\theta\right)-N_{s}(t) \sin \left(\omega_{0} t+\theta\right)\right]
$$

where

$$
\begin{aligned}
& N_{c}(t)=n_{c}(t) \cos (\theta-\varphi)+n_{s}(t) \sin (\theta-\varphi) \\
& N_{s}(t)=-n_{c}(t) \sin (\theta-\varphi)+n_{s}(t) \cos (\theta-\varphi)
\end{aligned}
$$

and

$$
N_{c}(t)+j N_{s}(t)=\left[n_{c}(t)+j n_{s}(t)\right] e^{-j(\theta-\phi)}
$$

The quadrature components $N_{c}(t)$ and $N_{s}(t)$ have exactly the same statistical characteristics as $n_{c}(t)$ and $n_{s}(t)$.

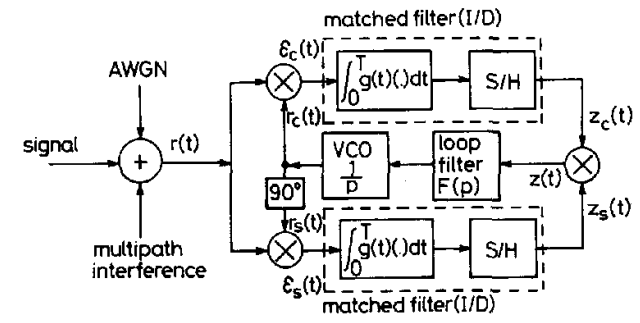

Fig. 2 I-Q carrier recovery loop for BPSK signal

A conventional maximum-likelihood (ML) phaserecovery loop for BPSK signal is shown in Fig. 2 [10]. The loop input is composed of the signal component, the multipath interference and the channel noise. The quadrature reference signals generated from the voltagecontrolled oscillator (VCO) can be written, respectively, by

$$
\begin{aligned}
& r_{c}(t)=\sqrt{ }(2) K_{1} \cos \left(\omega_{0} t+\hat{\theta}(t)\right) \\
& r_{s}(t)=\sqrt{ }(2) K_{1} \sin \left(\omega_{0} t+\hat{\theta}(t)\right)
\end{aligned}
$$

where $\theta(t)$ and $K_{1}$ represent the VCO estimate for the signal phase and the root mean square (RMS) value of the reference signals, respectively. In the usual way, the phase error is defined as

$$
\phi(t)=\theta-\theta(t)
$$

which is assumed to be constant over each $T$-second interval. In the sequel, to make the problem mathematically tractable, we assume that frequency offset is negligible and only phase uncertainty is considered. By using eqns. 6, 8 and 11, the phase detector (PD) outputs in the $I / Q$ channels can be arranged into $\cos \phi$ and $\sin \phi$ terms, neglecting the double frequency components, as

$$
\begin{aligned}
\varepsilon_{c}(t)= & K_{1} K_{m}\left\{\alpha \beta \sqrt{ }(P) m(t-\tau) \sin \left(-\omega_{F} \tau\right)+N_{c}(t)\right\} \\
& \times \cos \phi+K_{1} K_{m}\{\alpha \sqrt{ }(P) m(t)-\alpha \beta \sqrt{ }(P) m(t-\tau) \\
& \left.\times \cos \left(\omega_{F} \tau\right)-N_{s}(t)\right\} \sin \phi \\
\varepsilon_{s}(t)= & K_{1} K_{m}\{\alpha \sqrt{ }(P) m(t)-\alpha \beta \sqrt{ }(P) m(t-\tau) \\
& \left.\times \cos \left(\omega_{F} \tau\right)-N_{s}(t)\right\} \cos \phi+K_{1} K_{m} \\
& \times\left\{\alpha \beta \sqrt{ }(P) m(t-\tau) \sin \left(\omega_{F} \tau\right)-N_{c}(t)\right\} \sin \phi
\end{aligned}
$$

where $K_{m}$ is the gain of the loop multiplier, with dimension $V^{-1}$. On the other hand, the respective signal at the I/D filter output through a sample-and-hold (S/H) device 
can be described by

$$
\begin{aligned}
z_{c}(t) \equiv & \int_{0}^{T} g(t) \varepsilon_{c}(t) d t \\
= & -K_{1} K_{m} \alpha \beta \sqrt{ }(P) T\left\{\sin \left(\omega_{F} \tau\right) \cos \phi\right. \\
& \left.+\cos \left(\omega_{F} \tau\right) \sin \phi\right\} \sum_{k=0}^{L_{m}} a_{k} Q_{k} \\
& +K_{1} K_{m}\left\{\left(\alpha \sqrt{ }(P) T \sum_{k=0}^{L_{i}} a_{k} I_{k}-N_{1}\right)\right. \\
& \left.\times \sin \phi+N_{2} \cos \phi\right\} \\
z_{s}(t) \equiv & \int_{0}^{T} g(t) \varepsilon_{s}(t) d t \\
= & -K_{1} K_{m} \alpha \beta \sqrt{ }(P) T\left\{\cos \left(\omega_{F} \tau\right) \cos (\phi)\right. \\
& \left.-\sin \left(\omega_{F} \tau\right) \sin \phi\right\} \sum_{k=0}^{L_{m}} a_{k} Q_{k} \\
& +K_{1} K_{m}\left\{\left(\alpha \sqrt{ }(P) T \sum_{k=0}^{L_{i}} a_{k} I_{k}-N_{1}\right)\right. \\
& \left.\times \cos (\phi)-N_{2} \sin \phi\right\}
\end{aligned}
$$

where

$$
\begin{aligned}
I_{k} & =\frac{1}{T} \int_{0}^{T} g(t) p(t-k T) d t \\
Q_{k} & =\frac{1}{T} \int_{0}^{T} g(t) p(t-k T-\tau) d t
\end{aligned}
$$

and,

$$
\begin{aligned}
& N_{1} \equiv \int_{0}^{T} g(t) N_{s}(t) d t \\
& N_{2} \equiv \int_{0}^{T} g(t) N_{c}(t) d t
\end{aligned}
$$

The noise responses $N_{1}$ and $N_{2}$ at the I/D filter output are independent Gaussian random variables with identical variances $\sigma^{2}=N_{0} T / 2$. The parameters $L_{m}$ and $L_{i}$ in eqn. 14 represent the memory lengths of the bandlimited channel with and without multipath effect, respectively. The quantities $I_{k}$ and $Q_{k}$ in eqn. 15 can be easily calculated once $g(t)$ and $h(t)$ are known. The power of the signal baseband pulse is unity, i.e. $(1 / T) \int g^{2}(t) d t=1$. In the interval $k T \leqslant t<(k+1) T$, the normalised dynamic error signal $z^{\prime}(t)$ at the input of loop filter can be expressed as

$$
\begin{aligned}
z^{\prime}(t) \equiv & \frac{1}{\left(K_{1} K_{m}\right)^{2}} z(t)=\frac{1}{\left(K_{1} K_{m}\right)^{2}} z_{c}(t) z_{s}(t) \\
= & \left\{\frac{\alpha^{2} T^{2} P}{2}\left(\sum_{k=0}^{L_{i}} a_{k} I_{k}\right)^{2}-N_{1} T \alpha \sqrt{ }(P) \sum_{k=0}^{L_{i}} a_{k} I_{k}\right. \\
& \left.+\frac{N_{1}^{2}}{2}-\frac{N_{2}^{2}}{2}\right\} \sin 2 \phi \\
& +\left\{N_{2}\left[\alpha T \sqrt{ }(P) \sum_{k=0}^{L_{i}} a_{k} I_{k}-N_{1}\right]\right\} \cos 2 \phi \\
& +\frac{1}{2} \alpha^{2} \beta^{2} P T^{2} \sin \left(2 \phi+2 \omega_{F} \tau\right)\left(\sum_{k=0}^{L_{m}} a_{k} Q_{k}\right)^{2} \\
& -\alpha^{2} \beta P T^{2} \sin \left(2 \phi+\omega_{F} \tau\right)\left(\sum_{k=0}^{L_{i}} a_{k} I_{k}\right)\left(\sum_{k=0}^{L_{m}} a_{k} Q_{k}\right)
\end{aligned}
$$

$$
\begin{aligned}
& +\alpha \beta \sqrt{ }(P) T N_{1} \sin \left(2 \phi+\omega_{F} \tau\right)\left(\sum_{k=0}^{L_{m}} a_{k} Q_{k}\right) \\
& -\alpha \beta \sqrt{ }(P) T N_{2} \cos \left(2 \phi+\omega_{F} \tau\right)\left(\sum_{k=0}^{L_{m}} a_{k} Q_{k}\right)
\end{aligned}
$$

The low-frequency component (near DC) of $z^{\prime}(t)$ is extracted by the loop filter and used to adjust the phase estimate. By using linear model analysis [8-10], we adopt the approximation $\sin 2 \phi \simeq 2 \phi^{*}$, the phase estimate at the VCO output can be written as

$$
\theta(t)=\frac{1}{p} K_{v} F(p) z(t)
$$

where $p$ is the Heaviside operator defined by $p=d / d t$ and $K_{v}$ is the gain constant of VCO with dimension rad/ error is written as

$2 p \phi=-2 K_{v} F(p) z(t)=-2 K^{\prime} F(p) z^{\prime}(t)$

with

$$
K^{\prime}=K_{v}\left(K_{1} K_{m}\right)^{2}
$$

where $F(p)$ is the transfer function of the loop filter. Substituting eqn. 17 into eqn. 19 , after some mathematical manipulation the loop equation can be rearranged as

$$
2 p \phi+K^{\prime} P T^{2} F(p) x(t, 2 \phi) \sin 2 \phi=K^{\prime} F(p) N_{e}(t, 2 \phi)
$$

where

$$
\begin{aligned}
x(t, 2 \phi) \equiv & \alpha^{2}\left(\sum_{k=0}^{L_{k}} a_{k} I_{k}\right)^{2} \\
& +\alpha^{2} \beta^{2} \cos \left(2 \omega_{F} \tau\right)\left(\sum_{k=0}^{L_{m}} a_{k} Q_{k}\right)^{2} \\
& -2 \alpha^{2} \beta \cos \left(\omega_{F} \tau\right)\left(\sum_{k=0}^{L_{i}} a_{k} I_{k}\right)\left(\sum_{k=0}^{L_{m}} a_{k} Q_{k}\right)
\end{aligned}
$$

and

$$
N_{e}(t, 2 \phi)=N_{e}^{c}(t) \cos 2 \phi+N_{e}^{s}(t) \sin 2 \phi
$$

with

$$
\begin{aligned}
& N_{e}^{s}(t)=-2 \alpha \beta \sqrt{ }(P) T\left[N_{1} \cos \omega_{F} \tau\right. \\
& \left.+N_{2} \sin \omega_{F} \tau\right]\left(\sum_{k=0}^{L_{m}} a_{k} Q_{k}\right) \\
& +2 \alpha \sqrt{ }(P) T N_{1}\left(\sum_{k=0}^{L_{4}} a_{k} I_{k}\right)+\left(N_{2}^{2}-N_{1}^{2}\right) \\
& N_{e}^{c}(t)=-\alpha^{2} \beta^{2} P T^{2} \sin \left(2 \omega_{F} \tau\right)\left(\sum_{k=0}^{L_{m}} a_{k} Q_{k}\right)^{2} \\
& +2 \alpha^{2} \beta P T^{2} \sin \left(\omega_{F} \tau\right)\left(\sum_{k=0}^{L_{1}} a_{k} I_{k}\right)\left(\sum_{k=0}^{L_{m}} a_{k} Q_{k}\right) \\
& +2 \alpha \beta \sqrt{ }(P) T\left[N_{2} \cos \omega_{F} \tau-N_{1} \sin \omega_{F} \tau\right] \\
& \times\left(\sum_{k=0}^{L_{m}} a_{k} Q_{k}\right)-2 \alpha \sqrt{(P) T N_{2}}\left(\sum_{k=0}^{L_{i}} a_{k} I_{k}\right) \\
& +2 N_{1} N_{2}
\end{aligned}
$$
$\mathrm{s} / \mathrm{V}$. Referring to Fig. 2, the system equation for the phase 
The term $x(t, 2 \phi)$ conveys all the information regarding the phase of the received signal. The terms $N_{e}(t, 2 \phi)$ consituting the channel noises and other impairments are referred to as equivalent noise. From eqn. 22 , it can be found that there are self noises induced from the random terms $\left(\sum a_{k} I_{k}\right)^{2}, \quad\left(\sum a_{k} Q_{k}\right)^{2}$ and $\left(\sum a_{k} I_{k}\right)\left(\sum a_{k} Q_{k}\right)$ in $x(t, 2 \phi)$. Since these self-noise components are proportional to sin $2 \phi$, they can be neglected in the linear analysis for small phase error. As discussed in Reference 10 , the signal at the loop filter input can be approximated by the expectation value of the term $x(t, 2 \phi)$ over $\left\{a_{k}\right\}$. The effective signal amplitude is

$$
\begin{aligned}
A_{e} & \left.\equiv \frac{d}{d(2 \phi)}\left\{P T^{2} E[x(t, 2 \phi)] \sin 2 \phi\right\}\right|_{\phi: \text { small }} \\
& =P T^{2} E[x(t, 2 \phi)]
\end{aligned}
$$

where $E[x(t, 2 \phi)]$ is assumed to be independent of the time index $k$. The baseband linear model of the synchroniser in Fig. 2 is shown in Fig. 3. Using linear loop

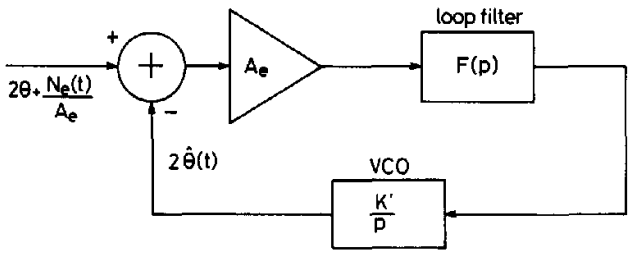

Fig. 3 Linear baseband model of synchroniser in Fig. 2

analysis, the small phase error $2 \phi$ can be expressed in terms of the transfer function by

$$
\begin{aligned}
2 \phi= & {\left[\frac{K^{\prime} P T^{2} F(p) E\{x(t, 2 \phi)\}}{p+K^{\prime} P T^{2} F(p) E\{x(t, 2 \phi)\}}\right] } \\
& \times\left[\frac{N(t)}{P T^{2} E\{x(t, 2 \phi)\}}\right]
\end{aligned}
$$

where $N(t)$ is the equivalent noise for small phase error, that is,

$$
N(t) \equiv N_{e}(t, 0)=N_{e}^{c}(t)
$$

and the expectation value of $x(t, 2 \phi)$ can be further manipulated and expressed as

$$
\begin{aligned}
\gamma \equiv & E\{x(t, 2 \phi)\} \\
= & \alpha^{2}\left(\sum_{k=0}^{L_{k}} I_{k}^{2}\right)+\alpha^{2} \beta^{2} \cos \left(2 \omega_{F} \tau\right)\left(\sum_{k=0}^{L_{m}} Q_{k}^{2}\right) \\
& -2 \alpha^{2} \beta \cos \left(\omega_{F} \tau\right)\left(\sum_{k=0}^{\min \left(L_{i}, L_{m}\right)} I_{k} Q_{k}\right)
\end{aligned}
$$

Define $\Gamma \equiv \gamma / \alpha^{2}$ to normalise $\gamma$ to the amplitude factor $\alpha^{2}$. For shorthand notation, the closed-loop transfer function for the phase error can be denoted by

$$
H_{2 \phi}(p) \equiv \frac{K^{\prime} P T^{2} F(p) E\{x(t, 2 \phi)\}}{p+K^{\prime} P T^{2} F(p) E\{x(t, 2 \phi)\}}
$$

The mean value of $2 \phi$ can be obtained from eqn. 26 as

$$
\mu_{2 \phi} \equiv E\{2 \phi\}=H_{2 \phi}(0) E\left\{\frac{N(t)}{P T^{2} E\{x(t, 2 \phi)\}}\right\}
$$

where

$$
\begin{aligned}
E\{N(t)\}= & -\alpha^{2} \beta^{2} P T^{2} \sin \left(2 \omega_{F} \tau\right)\left(\sum_{k=0}^{L_{m}} Q_{k}^{2}\right) \\
& +2 \alpha^{2} \beta P T^{2} \sin \left(\omega_{F} \tau\right)\left(\sum_{k=0}^{m i n} L_{i,}^{\left.L_{i}, L_{m}\right)} I_{k} Q_{k}\right)
\end{aligned}
$$

and $H_{2 \phi}(0)=1$. After some mathematical manipulation of eqns. 30 and 31 , the mean (averaged) value of phase error can be expressed as

$$
\begin{gathered}
-\beta^{2} \sin \left(2 \omega_{F} \tau\right)\left(\sum_{k=0}^{L_{m}} Q_{k}^{2}\right) \\
\mu_{2 \phi}=\frac{+2 \beta \sin \left(\omega_{F} \tau\right)\left(\sum_{k=0}^{\min \left(L_{k}, L_{m}\right)} I_{k} Q_{k}\right)}{\left(\sum_{k=0}^{L_{1}} I_{k}^{2}\right)+\beta^{2} \cos \left(2 \omega_{F} \tau\right)\left(\sum_{k=0}^{L_{m}} Q_{k}^{2}\right)} \\
-2 \beta \cos \left(\omega_{F} \tau\right)\left(\sum_{k=0}^{\min \left(L_{k}, I_{m}\right)} I_{k} Q_{k}\right)
\end{gathered}
$$

or

$$
\begin{aligned}
\mu_{2 \phi}= & \frac{1}{\Gamma}\left\{-\beta^{2} \sin \left(2 \omega_{F} \tau\right)\left(\sum_{k=0}^{L_{m}} Q_{k}^{2}\right)\right. \\
& +2 \beta \sin \left(\omega_{F} \tau\right)\left(\sum_{k=0}^{m i n}\left(L_{\left.i, L_{m}\right)} I_{k} Q_{k}\right)\right\}
\end{aligned}
$$

From eqn. 32, it is very obvious that $\mu_{2 \phi}$ is an odd function of the phase $\omega_{F} \tau$. Except for some special combination of $\omega_{\mathrm{F}} \tau$ and $\beta$ such that $\mu_{2 \phi}=0$, the multipath interference makes $\hat{\theta}$ be a biased estimate of the signal phase $\theta$. That is, the process $\hat{\theta}$ will converge to a steady state with an offset $\frac{1}{2} \mu_{2 \phi}$ from $\theta$. The excess phase offset is determined only by the multipath channel parameters and the ISI (BT parameter), and is independent of the additive white Gaussian noise. It cannot be reduced even if the loop bandwidth becomes very small. As $\beta$ approaches zero, i.e. multipath-free channel, the excess phase offset becomes zero

$$
\lim _{\beta \rightarrow 0} \mu_{2 \phi}=0
$$

That is, $\theta$ is an unbiased estimate for $\theta$ at the absence of multipath fading.

Now, we find the phase error variance. Instead of using $\sigma_{2 \phi}^{2}=E\left\{(2 \phi)^{2}\right\}-\left\{\mu_{2 \phi}\right\}^{2}$ relation, we adopt the linear transfer function approach. First, define the zeromean noise process $N^{\prime}(t)$ as $N^{\prime}(t)=N(t)-E\{N(t)\}$. Thus, the variance $\sigma_{2 \phi}^{2}$ is equivalent to the output power of $H_{2 \phi}(p)$ with $N^{\prime}(t)$ as input. Assuming that the loop bandwidth for the transfer function in eqn. 29 in the presence of ISI and multipath effects is still narrow relative to the bandwidth of the equivalent noise, then the variance of $2 \phi$ can be approximated $[10]$ by

$$
\sigma_{2 \phi}^{2} \simeq \frac{2 S_{N^{\prime}}(0) B_{L}}{A_{e}^{2}}=\frac{2 S_{N^{\prime}}(0) B_{L}}{P^{2} T^{4} E\{x(t, 2 \phi)\}^{2}}
$$

where the loop noise bandwidth $B_{L}$ is calculated by

$$
B_{L} \equiv \frac{1}{2 \pi j} \int_{0}^{j \infty}\left|H_{2 \phi}(s)\right|^{2} d s
$$

Owing to the sample-and-hold effect, the autocorrelation function $R_{N^{\prime}}(\tau)$ for $N^{\prime}(t)$, which is constant in each symbol

IEE PROCEEDINGS-I, VOl. 140, No. 5, OCTOBER 1993 
period, can be written $[6,10]$

$$
R_{N^{\prime}}(\tau)= \begin{cases}\sigma_{N}^{2}\left[1-\frac{|\tau|}{T}\right] & |\tau| \leqslant T \\ 0 & \text { otherwise }\end{cases}
$$

The Fourier transform of $R_{N^{\prime}}(\tau)$ appears as

$$
\begin{aligned}
S_{N^{\prime}}(\omega) & =\sigma_{N^{\prime}}^{2} \int_{-T}^{+T}\left(1-\frac{|\tau|}{T}\right) e^{-j \omega \tau} d \tau \\
& =\sigma_{N^{\prime}}^{2} T\left(\frac{\sin (\omega T / 2)}{\omega T / 2}\right)^{2}
\end{aligned}
$$

with

$$
S_{N^{\prime}}(0)=\sigma_{N^{\prime}}^{2} \int_{-T}^{+T}\left(1-\frac{|\tau|}{T}\right) d \tau=\sigma_{N^{\prime}}^{2} T
$$

where the noise variance $\sigma_{N}^{2}$ is defined as the expectation value of the squared equivalent noise process $N^{\prime}(t)$

$$
\begin{aligned}
\sigma_{N^{\prime}}^{2} \equiv & E\left\{N^{2}(t)\right\}=E\left\{N^{2}(t)\right\}-(E\{N(t)\})^{2} \\
= & \alpha^{4} \beta^{4} P^{2} T^{4} \sin ^{2}\left(2 \omega_{F} \tau\right)\left\{2\left(\sum_{k=0}^{L_{m}} Q_{k}^{2}\right)^{2}-2 \sum_{k=0}^{L_{m}} Q_{k}^{4}\right\} \\
& +4 \alpha^{4} \beta^{2} P^{2} T^{4} \sin ^{2}\left(\omega_{F} \tau\right)\left\{\left(\sum_{k=0}^{\min \left(L_{i,} L_{m}\right)} I_{k} Q_{k}\right)^{2}\right. \\
& \left.-2\left(\sum_{k=0}^{\min \left(L_{i,}, L_{m}\right)} I_{k}^{2} Q_{k}^{2}\right)+\left(\sum_{k=0}^{L_{i}} I_{k}^{2}\right)\left(\sum_{k=0}^{L_{m}} Q_{k}^{2}\right)\right\} \\
& +4 \alpha^{2} \beta^{2} P T^{2} \sigma^{2}\left(\sum_{k=0}^{L_{m}} Q_{k}^{2}\right)+4 \alpha^{2} P T^{2} \sigma^{2}\left(\sum_{k=0}^{L_{i}} I_{k}^{2}\right) \\
& -4 \alpha^{4} \beta^{3} P^{2} T^{4} \sin \left(2 \omega_{F} \tau\right) \sin \left(\omega_{F} \tau\right)\left\{2\left(\sum_{k=0}^{L_{m}} Q_{k}^{2}\right)\right. \\
& \left.\times\left(\sum_{k=0}^{\min \left(L_{i,} L_{m}\right)} I_{k} Q_{k}\right)-2\left(\sum_{k=0}^{\min \left(L_{i,} L_{m}\right)} I_{k} Q_{k}^{3}\right)\right\} \\
& -4 \alpha^{2} \beta P T^{2} \sigma^{2} \cos \left(\omega_{F} \tau\right)\left(\sum_{k=0}^{\min \left(L_{i,} L_{m}\right)} I_{k} Q_{k}\right)+4 \sigma^{4}
\end{aligned}
$$

where the expression for $E\{N(t)\}$ is given by eqn. 31 and that for $E\left\{N^{2}(t)\right\}$ is derived in the Appendix. Thus, the variance can be rearranged as

$$
\begin{aligned}
\sigma_{2 \phi}^{2}= & \frac{4 B_{L} T}{\Gamma^{2}}\left\{A_{1}\left[\left(\sum_{k=0}^{L_{m}} Q_{k}^{2}\right)^{2}-\sum_{k=0}^{L_{m}} Q_{k}^{4}\right]\right. \\
& +A_{2}\left[\left(\sum_{k=0}^{\min \left(L_{i,}, L_{m}\right)} I_{k} Q_{k}\right)^{2}-2\left(\sum_{k=0}^{\min \left(L_{i,}, L_{m}\right)} I_{k}^{2} Q_{k}^{2}\right)\right. \\
& \left.+\left(\sum_{k=0}^{L_{i}} I_{k}^{2}\right)\left(\sum_{k=0}^{L_{m}} Q_{k}^{2}\right)\right] \\
& +A_{3}\left[2\left(\sum_{k=0}^{L_{m}} Q_{k}^{2}\right)\left(\sum_{k=0}^{\min \left(L_{i,} L_{m}\right)} I_{k} Q_{k}\right)\right. \\
& \left.-2\left(\sum_{k=0}^{\min \left(L_{i,} L_{m}\right)} I_{k} Q_{k}^{3}\right)\right]+A_{4}\left(\sum_{k=0}^{L_{m}} Q_{k}^{2}\right) \\
& \left.+A_{5}\left(\sum_{k=0}^{L_{i}} I_{k}^{2}\right)+A_{6}\left(\sum_{k=0}^{\min \left(L_{L}, L_{m}\right)} I_{k} Q_{k}\right)+A_{7}\right\}
\end{aligned}
$$

where

$$
\begin{aligned}
& A_{1}=\beta^{4} \sin ^{2} 2 \omega_{F} \tau \\
& A_{2}=2 \beta^{2} \sin ^{2} \omega_{F} \tau
\end{aligned}
$$

$$
\begin{aligned}
& A_{3}=-2 \beta^{3} \sin 2 \omega_{F} \tau \sin \omega_{\mathrm{F}} \tau \\
& A_{4}=\frac{\beta^{2}}{\rho} \\
& A_{5}=\frac{1}{\rho} \\
& A_{6}=-\frac{\beta}{\rho} \cos \omega_{\mathrm{F}} \tau \\
& A_{7}=\frac{1}{2 \rho^{2}}
\end{aligned}
$$

and $\rho=\alpha^{2} P T / N_{0}$ defines the desired signal energy to noise density at the loop input. The analytical result in eqn. 41 relates the jitter variance performance to the effects of bandwidth limitation and multipath interference.

\section{Asymptotic bound}

Since the derivation of the analytic results in eqns. 32 and 41 is independent of the signalling pulse shape $g(t)$, they become the analytical bounds for the performance of carrier recovery loop in the bandlimited multipath channel. The most significant point of these analytic results is that they relate the first- and second-order statistical characteristics of the phase jitter to the impairments of ISI, multipath interference and channel noise.

In eqn. 41 , as signal-to-noise ratio $\rho$ in the multipath channel becomes large, the jitter variance becomes

$$
\begin{aligned}
\lim _{\rho \rightarrow \infty} \sigma_{2 \phi}^{2}= & \frac{4 B_{L} T}{\Gamma^{2}}\left\{A_{1}\left[\left(\sum_{k=0}^{L_{m}} Q_{k}^{2}\right)^{2}-\sum_{k=0}^{L_{m}} Q_{k}^{4}\right]\right. \\
& +A_{2}\left[\left(\sum_{k=0}^{m i n} I_{k}^{\left(L_{i}, L_{m}\right)} I_{k} Q_{k}\right)^{2}-2\left(\sum_{k=0}^{\min \left(L_{i,}, L_{m}\right)} I_{k}^{2} Q_{k}^{2}\right)\right. \\
& \left.+\left(\sum_{k=0}^{L_{j}} I_{k}^{2}\right)\left(\sum_{k=0}^{L_{m}} Q_{k}^{2}\right)\right]+A_{3}\left[2\left(\sum_{k=0}^{L_{m}} Q_{k}^{2}\right)\right. \\
& \left.\left.\times\left(\sum_{k=0}^{\min \left(L_{i,}, L_{m}\right)} I_{k} Q_{k}\right)-2\left(\sum_{k=0}^{\min \left(L_{i,}, L_{m}\right)} I_{k} Q_{k}^{3}\right)\right]\right\}
\end{aligned}
$$

where coefficients $\left\{A_{j}^{\prime}\right\}$ are defined in eqns. $42 a-c$. That is, even in the higher signal-to-noise case, the jitter variance is not zero. There exists a residual jitter variance determined by the ISI components from selective fading and band limiting. Since the values of coefficients $\left\{A_{j}^{\prime}\right\}$ are equal to zero as $\beta$ approaches zero, thus the residual jitter variance in eqn. 43 is also zero in the extreme case.

Furthermore, as the factor $\beta$ approaches zero, eqn. 41 becomes

$$
\lim _{\beta \rightarrow 0} \sigma_{2 \phi}^{2}=\frac{4 N_{0} B_{L}\left\{\alpha^{2} P T^{4}\left(\sum_{k=0}^{L_{i}} I_{k}^{2}\right)+\frac{1}{2} N_{0} T^{3}\right\}}{\alpha^{4} P^{2} T^{4}\left(\sum_{k=0}^{L_{i}} I_{k}^{2}\right)^{2}}
$$

or expressed as a function of $\rho$ by

$$
\lim _{\beta \rightarrow 0} \sigma_{2 \phi}^{2}=4 B_{L} T\left\{\frac{1}{\rho\left(\sum_{k=0}^{L_{i}} I_{k}^{2}\right)}+\frac{1}{2 \rho^{2}\left(\sum_{k=0}^{L_{i}} I_{k}^{2}\right)^{2}}\right\}
$$

The result in eqn. 44 is the same as developed in Reference 6 , where only ISI due to band-limiting is considered. 
The jitter variance, in this case, is determined by the ISI and the channel noise. Furthermore, if an ISI-free channel is considered, the jitter performance becomes that in Reference 10 (p. 255),

$$
\begin{aligned}
\lim _{\substack{l_{i} \rightarrow 0 \\
\mathcal{L}_{i} \rightarrow 0}} \sigma_{2 \phi}^{2} & =\frac{4 N_{0} B_{L}}{\alpha^{4} P^{2} T^{4}}\left\{\alpha^{2} P T^{4}+\frac{1}{2} N_{0} T^{3}\right\} \\
& =4 B_{\mathrm{L}} T\left(\frac{1}{\rho}+\frac{1}{2 \rho^{2}}\right)
\end{aligned}
$$

In this case, it is obvious that the performance of the carrier recovery loop is dominated by the channel noise.

\section{Numerical examples}

Without loss of generality, a fifth-order Butterworth lowpass filter is used as an example of the bandlimited channel. The ISI effect caused by bandlimitation can be controlled by the parameter $B T$, where $B$ is the $3 \mathrm{~dB}$ cut-off frequency. Fig. 4 shows a family of pulse responses for such an LPF with $B T=0.7,0.9$ and 1.1, respectively.

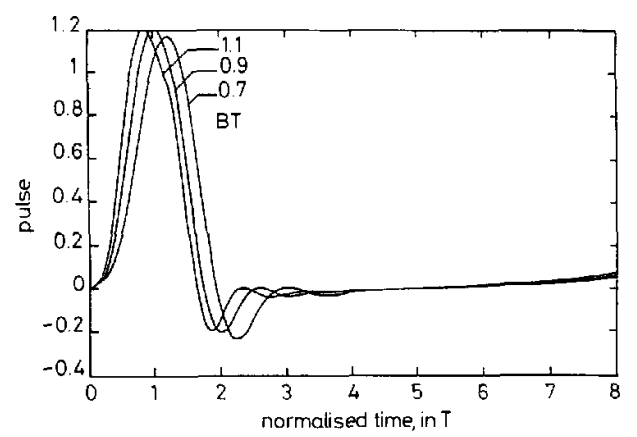

Fig. 4 Pulse responses of fifth-order Butterworth LPF $B T=0.7,0.9,1.1$

Once the multipath channel parameters are given by using the closed-form expression in eqn. 41 the jitter variance can be calculated numerically. Fig. 5 shows a numerical result of eqn. 41 , normalised to loop parameter $4 B_{L} T$, for a channel with severe fading, where the channel is described by the following model parameters: $\tau=6.31 \mathrm{~ns}, \alpha=0.0322, \beta=0.9010, f_{F}=5.78 \mathrm{MHz}^{*}$ and

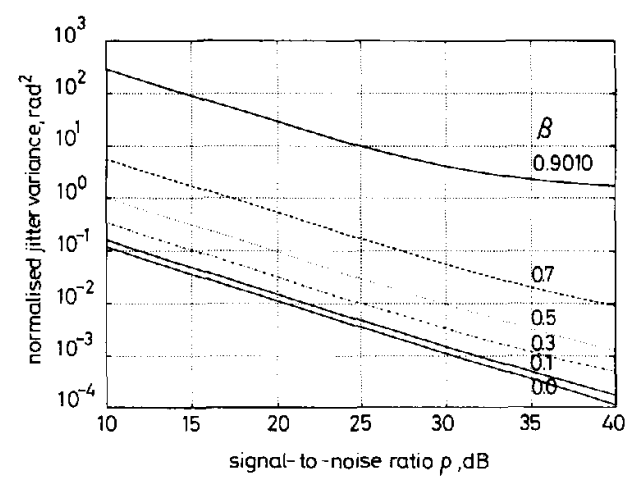

Fig. 5 Numerical results for normalised jitter variance $x=0.0322$, BT $=1.0, \tau=0.164 T, f_{F}=5.78 \mathrm{MHz}$

- Fitting parameters for measured fade in Reference 13 (Fig. 2)
$B T=1.0$. The relative notch depth, $B=-20 \log _{10}(1-\beta)$ is $20.1 \mathrm{~dB}$, the scale factor $A=-20 \log _{10} \alpha$, is $29.8 \mathrm{~dB}$, and the notch frequency is $5.78 \mathrm{MHz}$ above the central frequency. It is a severe fade both in shape and depth. In Fig. 5, to compare the effect of frequency selectivity, numerical calculation results for various values of $\beta$ are also shown $(\beta=0.7,0.5,0.3,0.1,0.0)$, where $\beta=0.0$ represents the case without multipath interference. The timing for the $/ / D$ filter is chosen at that the signal has minimum ISI

The numerical results shown in Fig. 5 are obtained with deterministic channel parameters. However, these parameters are usually random in practice, thereby it will be more realistic to examine the result for random quantities. In general, given the joint statistical distributions of $\alpha, \beta$ and $\omega_{F}$, the expectation value of the loop performance can be evaluated directly. For the sake of convenience, variable transformations are taken to ease the mathematical manipulation. Here, we introduce the new random variables $A, B$ and $\Psi$, which are related to the channel parameters $\alpha, \beta, \tau$ and $\omega_{F} \tau$ by

$$
\begin{aligned}
A & \equiv-20 \log _{10} \alpha \quad 0 \leqslant \alpha \leqslant 1 \\
B & \equiv-20 \log _{10}(1-\beta) \quad 0 \leqslant \beta \leqslant 1 \\
\Psi & \equiv 360 f_{F} \tau
\end{aligned}
$$

Define $p_{A B \Psi}(A, B, \Psi)$ as an arbitrary joint probability density function of $A, B$ and $\Psi$. Then the statistical averages of $\sigma_{2 \phi}^{2}$ and $\mu_{2 \phi}$ with respect to the channel statistics can be manipulated in a general form, respectively, by

$$
\begin{aligned}
& \bar{\sigma}_{2 \phi}^{2} \equiv E\left[\sigma_{2 \phi}^{2}\right]=\iiint p_{A B \Psi}(A, B, \Psi) \sigma_{2 \phi}^{2} d A d B d \Psi \\
& \bar{\mu}_{2 \phi} \equiv E\left[\mu_{2 \phi}\right]=\iiint p_{A B \Psi}(A, B, \Psi) \mu_{2 \phi} d A d B d \Psi
\end{aligned}
$$

In fact, the probability density functions of the channel parameters for Rummler's model have been well recognised [11]. Based on the statistics, an illustrative example for eqns. 48 and 49 is described in the following.

First, the choice of the parameter $\tau$ in the fixed delay model is dependent on the channel bandwidth. In Reference 11 , the choice of $\tau=6.31 \mathrm{~ns} \dagger$ provides the best fitting of the model from measurement data. The distribution of the relative strength factor $\beta$ can be written in exponential form $(1-\beta)^{2.3}$. The probability finding $\beta$ between 0.0 and 0.5 is $\operatorname{Prob}(0.0 \leqslant \beta \leqslant 0.5)=0.79$. Similarly, the probability Prob $(0.0 \leqslant \beta \leqslant 0.7)$ is 0.94 . That is, the small value of $\beta$ (less selective) occurs more frequently. On the other hand, the overall attenuation factor $\alpha$ is characterised with lognormal distribution. The standard deviation of the distribution is approximately $5 \mathrm{~dB}$ for all $\beta$. The mean of the distribution is dependent on the $\beta$ factor. For $\beta>0.5$, the mean value is close to $25 \mathrm{~dB}$. In contrast, for smaller value of $\beta$, the mean value decreases to $15 \mathrm{~dB}$. The probability density function of $f_{F}=\omega_{F} / 2 \pi$ is uniform at two levels and can be expressed as

$$
p\left(f_{F}\right)= \begin{cases}\frac{5 \tau}{3} & \left|f_{F}\right| \leqslant \frac{1}{4 \tau} \\ \frac{\tau}{3} & \frac{1}{4 \tau} \leqslant\left|f_{F}\right| \leqslant \frac{1}{2 \tau}\end{cases}
$$

In Reference 11, the radio channel was equipped with 8 PSK at a rate of $78 \mathrm{Mbit} / \mathrm{s}$ occupying $30 \mathrm{MHz}$ bandwidth. To keep the consistency of this channel condition (statistics) for BPSK, in the illustrative examples, transmission at a rate of $26 \mathrm{Mbit} / \mathrm{s}$ in the same bandwidth is assumed so that $\tau \simeq 0.164 T$ ( $T$ : bit period for BPSK).

IEE PROCEEDINGS-1, Vol. 140, No. 5, OCTOBER 1993 
where $\tau$ is the fixed delay. Taking the variable transformation in eqn. 47 , the statistical distributions of $A, B$ and $\Psi$ can be written, respectively, [12]

$$
p_{A}(A)=\frac{1}{\sqrt{ }(2 \pi) 5} e^{-\left(A-\mu_{A}\right)^{2} / 50}
$$

with

$$
\begin{aligned}
& \mu_{A}=24.6\left(B^{4}+500\right) /\left(B^{4}+800\right) \\
& p_{B}(B)=\frac{1}{3.8} e^{-B / 3.8} \\
& p_{\Psi}(\Psi)= \begin{cases}\frac{1}{216} & |\Psi| \leqslant 90^{\circ} \\
\frac{1}{1080} & 90^{\circ}<|\Psi|<180^{\circ}\end{cases}
\end{aligned}
$$

In particular, the distribution of $\Psi$ (or notch frequency) is independent of the other parameters. There exists dependence between parameters $A$ and $B$ as shown in eqn. 51, where the mean value of $A, \mu_{A}$, can be expressed in a simple form of $B$. Although the distribution of $A$ is dependent on the value of $B$, the dependence is limited and may often be ignored [13]. Introducing eqn. 51, the expectation value of the jitter variance in eqn. 48 can be written by

$$
\begin{aligned}
\bar{\sigma}_{2 \phi}^{2}= & \int_{-180}^{+180} p_{\Psi}(\Psi) \int_{0}^{\infty} \int_{0}^{\infty} p_{A}(A) p_{B}(B) \\
& \times \sigma_{2 \phi}^{2}(A, B, \Psi, \tau) d A d B d \Psi
\end{aligned}
$$

Fig. 6 shows the numerical results of $\bar{\sigma}_{2 \phi}^{2}$ (normalized to loop parameter $4 B_{L} T$ ) against $\rho$ with $B T$ as the parameters. It is found that the jitter increases with the reduction of $B T$ and the signal-to-noise ratio $\rho$ Reduction of $B T$ or $\rho$ will introduce more ISI or noise fluctuation, and thus, the jitter variance will increase. In a similar way, the expectation value of the excess phase offset $\left(\mu_{2 \phi}\right)$ in eqn. 49 can be evaluated by

$$
\begin{aligned}
\bar{\mu}_{2 \phi}= & \int_{-180}^{+180} p_{\Psi}(\Psi) \int_{0}^{\infty} \int_{0}^{\infty} p_{A}(A) p_{B}(B) \\
& \times \mu_{2 \phi}(A, B, \Psi, \tau) d A d B d \Psi
\end{aligned}
$$

Since the channel noise has no contribution to $\mu_{2 \phi}$ (eqn. 32 ), so $\overline{\mu_{2 \phi}}$ is independent of the signal-to-noise ratio $\rho$.

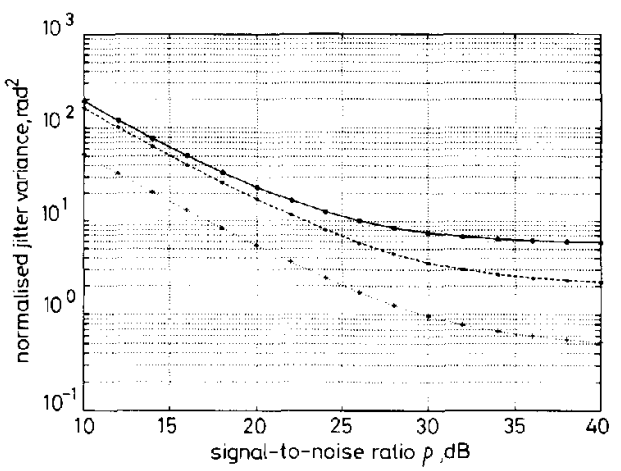

Fig. 6 Expectation value of normalised jitter variance against $S N R$ in the multipath fading channel

$\begin{array}{ll}O & B T \\ * \quad & B T=1.9 \\ +\quad B T & =1.1\end{array}$

IEE PROCEEDINGS-I, Vol. 140, No. 5, OCTOBER 1993
In fact, because $\mu_{2 \phi}$ is an odd function of $\Psi$ (see eqn. 32) and the distribution of $\Psi$ is even in $\left[-180^{\circ},+180^{\circ}\right], \overline{\mu_{2 \phi}}$ is equal to zero.

\section{Conclusions}

Closed-form expressions of the jitter variance and the excess phase error for I-Q carrier recovery loop with BPSK signal in the band-limited multipath channel are derived. The analytical bound can be evaluated by numerical calculation. From these results, the degradation of synchronizer performance due to multipath and ISI effects can be predicted directly. Further extension of the results for random channel parameters are also shown by numerical illustration.

\section{References}

1 RAGHAVON, S.H.R., and ZIEMER, R.F.: 'Improving QPSK transmission in band-limited channels with interference through equalization', IEEE Trans., 1977, COM-25, pp. 1222-1226

2 FALCONER, D.D.: 'Jointly adaptive equalization and carrier recovery in two-dimensional digital communications systems', Bell Syst. Tech. J., 1976, 55, pp. 317-334

3 FALCONER, D.D.: 'Analysis of a gradient algorithm for simultaneous passband equalization and carrier phase recovery', Bell Syst. Tech. J., 1976, 55, pp. 409-428

4 GODARD, D.M.: 'Self-recovering equalization and carrier tracking in two-dimensional data communication systems', IEEE Trans., 1980, COM-29, pp. 1867-1875

5 MORIDI, $S$, and SARI, $H$. "Analysis of four decision feedback carrier recovery loops in the presence of intersymbol interference', IEEE Trans., 1985, COM-33, pp. 543-550

6 HINEDI, S., and LINDSEY, W.C.: 'Intersymbol interference effects on BPSK and QPSK carrier tracking loops', IEEE Trans., 1990, COM-38, pp. 1670-1676

7 PROAKIS, J.G.: 'Digital communications' (McGraw-Hill, New York, USA, 1989)

8 GARDNER, F.M. 'Phaselock techniques' (Wiley, 1979, 2nd edn.)

8 GARDNER, F.M.: 'Phaselock techniques' (Wiley, 1979, 2nd edn.) engineering' (Prentice-Hall, Englewood Cliffs, New Jersey, USA, 1972)

10 STIFFLER, J.J.: 'Theory of synchronous communications' (Prentice-Hall, Englewood Cliffs, New Jersey, USA, 1971)

11 RUMMLER, W.D.: 'A new selective fading model: application to propagation data', Bell Syst., Tech. J., 1979, 58, pp. 1037-1071

12 LUNDGREN, C.W., and RUMMLER, W.D.: 'Digital radio outage due to selective fading-observation vs. prediction from laboratory simulation', Bell Syst., Tech. J., 1979, 58, pp. 1073-1100

13 RUMMLER, W.D.: 'More on the multipath fading channel model', IEEE Trans., 1981, COM-29, (3), pp. 346-352

14 RUMMLER, W.D.: 'Time- and frequency domain representation of multipath fading on line-of-sight microwave paths', Bell Syst. Tech. $J ., 1980,59,(5)$, pp. 763-796

15 LAM, W.I., and WEBSTER, A.R.: 'Microwave propagation on two line-of-sight oversea paths', IEEE Trans., 1985, AP-33, (5), pp. 510516

16 SAKAGAMI, S., and HOSOYA, Y.: 'Some experimental results on in-band amplitude dispersion and a method for estimating in-band linear amplitude dispersion', IEEE Trans., 1982, COM-30, (8), pp. $1875-1888$

17 LEE, T.C., and LIN, S.H.: 'More on frequency diversity for digital radio'. IEEE conference, Global communications, 1985, no. 3, pp. 1108-1112

18 SHAFI, M., and TAYLOR, D.P.: 'Influence of terrain induced reflections on the performance of high capacity digital radio
systems'. IEEE conference, International conference on Communications, 1986, no. 3, pp. 1627-1631

19 GOLDMAN, H.: "Mathematical analysis of the three-ray dispersive fading channel model', IEE Proc. I, 1991, 138, (2), pp. 87-94

20 BEST, R.E.: 'Phase-locked loops-theory, design and application' (McGraw-Hill, New York, 1984)

\section{Appendix}

The equivalent noise $N(t)$ for small phase error can be obtained from

$$
\begin{aligned}
N(t) & \equiv N_{e}(t, 2 \phi) @ \phi \simeq 0 \\
& =N_{e}^{c}(t)
\end{aligned}
$$


and

$$
\begin{aligned}
N_{e}^{c}(t)= & -\alpha^{2} \beta^{2} P T^{2} \sin \left(2 \omega_{F} \tau\right)\left(\sum_{k=0}^{L_{m}} \sum_{l=0}^{L_{m}} a_{k} a_{l} Q_{k} Q_{l}\right) \\
& +2 \alpha^{2} \beta P T^{2} \sin \left(\omega_{F} \tau\right)\left(\sum_{k=0}^{L_{l}} \sum_{l=0}^{L_{m}} a_{k} a_{l} I_{k} Q_{l}\right) \\
& +2 \alpha \beta \sqrt{ }(P) T\left(N_{2} \cos \left(\omega_{F} \tau\right)\right. \\
& -N_{1} \sin \left(\omega_{F} \tau\right)\left(\sum_{k=0}^{L_{m}} a_{k} Q_{k}\right) \\
& -2 \alpha \sqrt{ }(P) T N_{2}\left(\sum_{k=0}^{L_{l}} a_{k} I_{k}\right)+2 N_{1} N_{2}
\end{aligned}
$$

Note that $N_{1}$ and $N_{2}$ are two independent noise processes with zero-mean and identical variance $\sigma^{2}$. Performing the expectation operation on the squared equivalent noise, obtains

$$
\begin{aligned}
E\left\{N^{2}(t)\right\} & \\
= & \alpha^{4} \beta^{4} P^{2} T^{4} \sin ^{2}\left(2 \omega_{F} \tau\right) \\
& \times\left(\sum_{k=0}^{L_{m}} \sum_{l=0}^{L_{m}} \sum_{k^{\prime}=0}^{L_{m}} \sum_{l^{\prime}=0}^{L_{m}} E\left(a_{k} a_{l} a_{k^{\prime}} a_{l^{\prime}}\right) Q_{k} Q_{l} Q_{k^{\prime}} Q_{l^{\prime}}\right) \\
& -4 \alpha^{4} \beta^{3} P^{2} T^{4} \sin \left(2 \omega_{F} \tau\right) \sin \left(\omega_{F} \tau\right) \\
& \times\left(\sum_{k=0}^{L_{m}} \sum_{l=0}^{L_{m}} \sum_{k^{\prime}=0}^{L_{4}} \sum_{l^{\prime}=0}^{L_{m}} E\left\{a_{k} a_{l} a_{k^{\prime}} a_{l^{\prime}}\right\} Q_{k} Q_{l} I_{k^{\prime}} Q_{l^{\prime}}\right) \\
& +4 \alpha^{4} \beta^{2} P^{2} T^{4} \sin ^{2}\left(\omega_{F} \tau\right) \\
& \times\left(\sum_{k=0}^{L_{i}} \sum_{l=0}^{L_{m}} \sum_{k^{\prime}=0}^{L_{l}} \sum_{l^{\prime}=0}^{L_{m}} E\left\{a_{k} a_{l} a_{k^{\prime}} a_{l^{\prime}}\right\} I_{k} Q_{l} I_{k^{\prime}} Q_{l^{\prime}}\right) \\
& +4 \alpha^{2} \beta^{2} P T^{2} E\left\{\left[N_{2} \cos \left(\omega_{0} \tau\right)+N_{1} \sin \left(\omega_{0} \tau\right)\right]^{2}\right\} \\
& \times\left(\sum_{k=0}^{L_{m}} \sum_{l=0}^{L_{m}} E\left\{a_{k} a_{l}\right\} Q_{k} Q_{l}\right)+4 \alpha^{2} \beta^{2} P T^{2} \\
& \times E\left\{\left[N_{2} \cos \left(\omega_{F} \tau\right)-N_{1} \sin \left(\omega_{F} \tau\right)\right]^{2}\right\} \\
& \times\left(\sum_{k=0}^{L_{m}} \sum_{l=0}^{L_{m}} E\left\{a_{k} a_{l}\right\} Q_{k} Q_{l}\right)-4 \alpha^{2} \beta P T^{2}
\end{aligned}
$$

$$
\begin{aligned}
& \times \cos \left(\omega_{F} \tau\right) E\left\{N_{2}^{2}\right\}\left(\sum_{k=0}^{L_{1}} \sum_{l=0}^{L_{m}} E\left\{a_{k} a_{l}\right\} I_{k} Q_{l}\right) \\
& +4 \alpha^{2} P T^{2} E\left\{N_{2}^{2}\right\}\left(\sum_{k=0}^{L_{1}} \sum_{l=0}^{L_{i}} E\left\{a_{k} a_{l}\right\} I_{k} I_{l}\right) \\
& +4 E\left\{N_{1}^{2}\right\} E\left\{N_{2}^{2}\right\}
\end{aligned}
$$

By using the following relations,

$$
\begin{aligned}
& E\left\{N_{1}^{2}\right\}=E\left\{N_{2}^{2}\right\}=\sigma^{2} \\
& E\left\{a_{k} a_{l}\right\}=\delta_{k l}
\end{aligned}
$$

where $\delta_{k l}$ denotes the Kronecker delta function, the higher-order moment

$$
\begin{aligned}
& E\left\{a_{k} a_{l} a_{k^{\prime}} a_{l^{\prime}}\right\}=\delta_{k l} \delta_{k^{\prime} t^{\prime}}+\delta_{k k^{\prime}} \delta_{l l^{\prime}} \\
& +\delta_{k l^{\prime}} \delta_{l k^{\prime}}-2 \delta_{k l} \delta_{l k^{\prime}} \delta_{k^{\prime} t^{\prime}} \\
& \sum_{k=0}^{L_{m}} \sum_{l=0}^{L_{m}} \sum_{k^{\prime}=0}^{L_{m}} \sum_{l^{\prime}=0}^{L_{m}} E\left\{a_{k} a_{l} a_{k^{\prime}} a_{l^{\prime}}\right\} Q_{k} Q_{l} Q_{k^{\prime}} Q_{l^{\prime}} \\
& =3\left(\sum_{k=0}^{L_{m}} Q_{k}^{2}\right)^{2}-2\left(\sum_{k=0}^{L_{m}} Q_{k}^{4}\right)
\end{aligned}
$$

we obtain

$\sum_{k=0}^{L_{l}} \sum_{l=0}^{L_{m}} \sum_{k^{\prime}=0}^{L_{l}} \sum_{l^{\prime}=0}^{L_{m}} E\left\{a_{k} a_{l} a_{k^{\prime}}, a_{l^{\prime}}\right\} I_{k} Q_{l} I_{k^{\prime}}, Q_{l^{\prime}}$

$$
\begin{aligned}
= & 2\left(\sum_{k=0}^{\min \left(L_{k}, L_{m}\right)} I_{k} Q_{k}\right)^{2}+\left(\sum_{k=0}^{L_{k}} I_{k}^{2}\right)\left(\sum_{k=0}^{L_{m}} Q_{k}^{2}\right) \\
& -2\left(\sum_{k=0}^{\min \left(L_{k}, L_{m}\right)} I_{k}^{2} Q_{k}^{2}\right)
\end{aligned}
$$

$\sum_{k=0}^{L_{m}} \sum_{l=0}^{L_{m}} \sum_{k^{\prime}=0}^{L_{l}} \sum_{l^{\prime}=0}^{L_{m}} E\left\{a_{k} a_{l} a_{k^{\prime}} a_{l^{\prime}}\right\} Q_{k} Q_{l} I_{k^{\prime}} Q_{l^{\prime}}$

$$
\begin{aligned}
= & 3\left(\sum_{k=0}^{L_{m}} Q_{k}^{2}\right)\left(\sum_{k=0}^{m i n} I_{k} L_{k} L_{m}\right) \\
& -2\left(\sum_{k=0}^{\min \left(L_{i}, L_{m}\right)} I_{k} Q_{k}^{3}\right)
\end{aligned}
$$

Combining eqns. 56-62 and 31, the final formula in eqn. 40 is obtained. 